\title{
GENERALIZATION OF WEIGHTED OSTROWSKI TYPE INEQUALITIES BY ABEL-GONTSCHAROFF POLYNOMIAL
}

\author{
Andrea Aglić Aluinović, LJiljanka KVEsić, Josip PeČArić \\ AND SANJA TIPURIĆ-SPUŽEVIĆ
}

Abstract. We present a weighted generalization of Ostrowski type inequality for continous functions presented by Abel-Gontscharoff interpolating polynomial

Mathematics subject classification (2010): 26D15, 26D20.

Keywords and phrases: Ostrowski type inequality, Abel-Gontscharoff polynomials.

\section{REFERENCES}

[1] R. P. Agarwal, P. J. Y. Wong, Error Inequalities in Polynomial Interpolation and Their Applications, Kluwer Academic Publishers, Dordrecht/Boston/London, 1993.

[2] A. Aglić Aluinović, A note on generalization of weighted Čebyšev and Ostrowski inequalities, J. Math. Inequal. 3, 3 (2009), 409-416.

[3] A. Aglić Aluinović, J. PeČARIĆ, On some Ostrowski type inequalities via Montgomery identity and Taylor's formula, Tamkang J. Math. 36, 3 (2005), 199-218.

[4] A. Aglić Aluinović, J. PečArić, I. Perić, Estimates of the difference between two weighted integral means via weighted Montgomery identity, Math. Inequal. Appl. 7, 3 (2004), 315-336.

[5] A. Aglić Aljinović, J. Pečarić, A. Vukelić, On some Ostrowski type inequalities via Montgomery identity and Taylor's formula II, Tamkang J. Math. 36, 4 (2005), 279-301.

[6] A. Aglić Aljinović, A. ČIVluak, S. Kovač, J. PeČARić, M. Ribičić Penava, General Integral Identities and Related inequalities, Element, Zagreb, 2013.

[7] D. S. Mitrinović, J. E. PeČARIĆ, AND A. M. Fink, Inequalities for functions and their Integrals and Derivatives, Kluwer Academic Publishers, Dordrecht, 1994.

[8] A. Ostrows KI, Über die Absolutabweichung einer differentiebaren Funktion von ihrem Integralmittelwert, Comment. Math. Helv. 10 (1938), 226-227.

[9] J. PEČARIĆ, On the Čebyšev inequality, Bul. Inst. Politehn. Timisoara 25 (39) (1980), 10-11. 